\title{
Momentum-resolved EELS measurements of hexagonal boron nitride
}

\author{
R. Arenal *, M. Kociak**, N.J. Zaluzec* \\ * EMC group, MSD Argonne National Laboratory, IL 60439 Argonne - USA \\ ** LPS, Université Paris-Sud and CNRS, 91405 Orsay - France
}

Hexagonal boron nitride (h-BN) is an insulating material structurally similar to semimetallic graphite. In recent years, the interest of h-BN has increased due to the development of techniques to synthesize boron nitride nanotubes (BNNTs). Fig. 1 shows a high resolution TEM image of singlewalled BNNTs synthesized at ONERA (France) using laser vaporization technique [1]. Structurally, these nanotubes can be envisioned as an h-BN sheet rolled up into a cylinder. In order to understand the related properties in BNNTs, an improved knowledge of the physical properties of $\mathrm{h}-\mathrm{BN}$ is necessary $[1,2]$. In our work we are using electron energy loss spectroscopy (EELS) in a transmission electron microscope to accomplish some of this task. By studying both the core loss and low loss regions of an EEL spectrum we are able to derive complementary information on the electronic structure and dielectric response of BN. In the specific case of an anisotropic crystal, like h-BN, EELS we are employing angle-resolved experiments allow to probe this anisotropy in the electronic structure.

Our angle-resolved EELS (core and low-loss regions) measurements on highly pure h-BN microcrystals were obtained using the High Angle Resolved Electron Channelling Spectroscopy (HARECES) technique [3]. We performed this work in a FEI Tecnai F-20 electron microscope, equipped with a GIF and operating at $200 \mathrm{kV}$. We employed a small collection aperture $(\beta)$ of $<0.3$ mrad together with a highly coherent and nearly parallel probe $(\alpha<0.05 \mathrm{mrad})$. The diameter of this probe used during these measurements was typically one micron which was used to define the region of interest on a uniformly thick single crystal region of an oriented grain. Measurements were done on or near the $\{0001\}$ axis as can be seen in the selected area diffraction pattern shown in Fig. 1 (c). Prior to analysis the sample was cleaned for 15 minutes in Argon using a SouthBay Technology PC2000 plasma cleaner to mitigate the deleterious effects of hydrocarbon contamination which was not detectable during the course of our temporally extended measurements.

The high resolution measurements of the electronic states of boron and nitrogen were performed by measuring the fine structure of ionization edges (ELNES) in order to extend the range of information delivered by conventional EELS experiments. Previous work on h-BN, achieved by EELS: in the low-loss region [2,4,5], in the core loss region [6] and also by x-ray Raman spectroscopy [7] has pointed out that there is extreme anisotropy in the electronic structure of this material. Our results, augmented by theoretical calculations [8], significantly improve on the older work. Examples of experimental B-K and N-K edges are shown in Fig. 2 for three different scattering vectors along the momentum transfer direction of $<11-20>$. Two of them (coloured blue and red) correspond to the two extreme orientations with the momentum transfer $(\mathbf{q})$ parallel and perpendicular to the c-axis, respectively. As one can see the fine structure of these K edges depend strongly on the orientation of the scattering vector with respect to the lattice. The first peak of the boron and nitrogen K edges disappears completely under the q perpendicular to $\mathbf{c}$ condition. This confirms that the origin of these peaks is the transition of a 1 s electron to the $\mathrm{B}-2 p_{z}$ and the $\mathrm{N}-2 p_{z}$ orbitals, in the boron and nitrogen edge respectively. Nevertheless, we note that in the B-K edge the origin of this first peak is excitonic while this is not the case for N-K edge. The differences between the spectra due to the 
anisotropy of the h-BN are also visible in the set of peaks in the spectra after the $\pi^{*}$ peak which correspond to the $\sigma^{*}$ resonances. In the B-K edge, the main of these differences concern: (i) the enhancement of the peak at $208 \mathrm{eV}$ for the orientation $\mathbf{q} / / \mathbf{c}$ and, (ii) from $220 \mathrm{eV}$ the different behavior (presence, of a peak around $225 \mathrm{eV}$ and absence of the broad peak centered at $230 \mathrm{eV}$ ) of the spectrum for the orientation $\mathbf{q}$ perpendicular to c comparing with the others. These differences ((i) and (ii)) are due to the influence of the different contributions, as a function of the orientation, of the core electrons transitions to the $\mathrm{B}-2 p_{x y}$ and $\mathrm{B}-2 p_{z}$ orbitals. As in the $\mathrm{BN}$, the bond between $\mathrm{B}$ and $\mathrm{N}$ atoms is relatively strong covalent, and thus the shape of the N-K edge is similar to this of the B-K edge. The interpretation of the different peaks for the N-K edge is therefore similar to that of B-K when one takes into account the $\mathrm{N}$ orbitals instead those of $\mathrm{B}$. In continuation of this work, we intend to perform similar measurements on the orientation dependence of the low loss profile.

\section{References}

[1] R. Arenal, PhD Thesis U. Paris XI - Orsay, 2005.

[2] R. Arenal, et al., Phys. Rev. Lett. 95, 127601 (2005).

[3] Zaluzec N.J. Microscopy Microanalysis Vol 6, Sup 2, 938 (1999)

[4] M.R. Vilanove, C. R. Acad. Scien. Ser. B 271, 1101 (1972).

[5] C. Tarrio, S.E. Schnatterly, Phys. Rev. B 40, 7852 (1989).

[6] R.D. Leapman, et al., Phys. Rev. B 28, 2361 (1983).

[7] N. Watanabe et al., Appl. Phys. Lett. 691370 (1996).

[8] I. Tanaka et al., Phys. Rev. B 60, 4944 (1999).

[9] This work was supported in part by the U.S. DOE under BES-MS W-31-109-Eng-38 at ANL

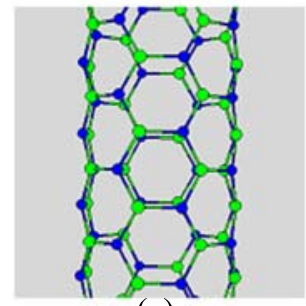

(a)

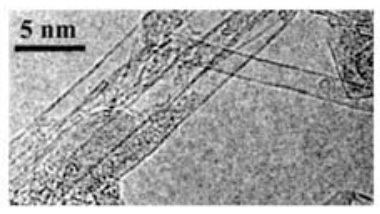

(b)

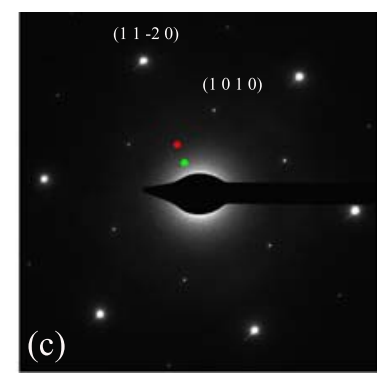

Figure 1. a) and b) schema and high resolution TEM image of SW-BNNTs, respectively. c) Selected area electron diffraction of a single-crystal of h-BN (the circles (coloured red and green) indicate the $\mathbf{q}$ transfer directions studied).
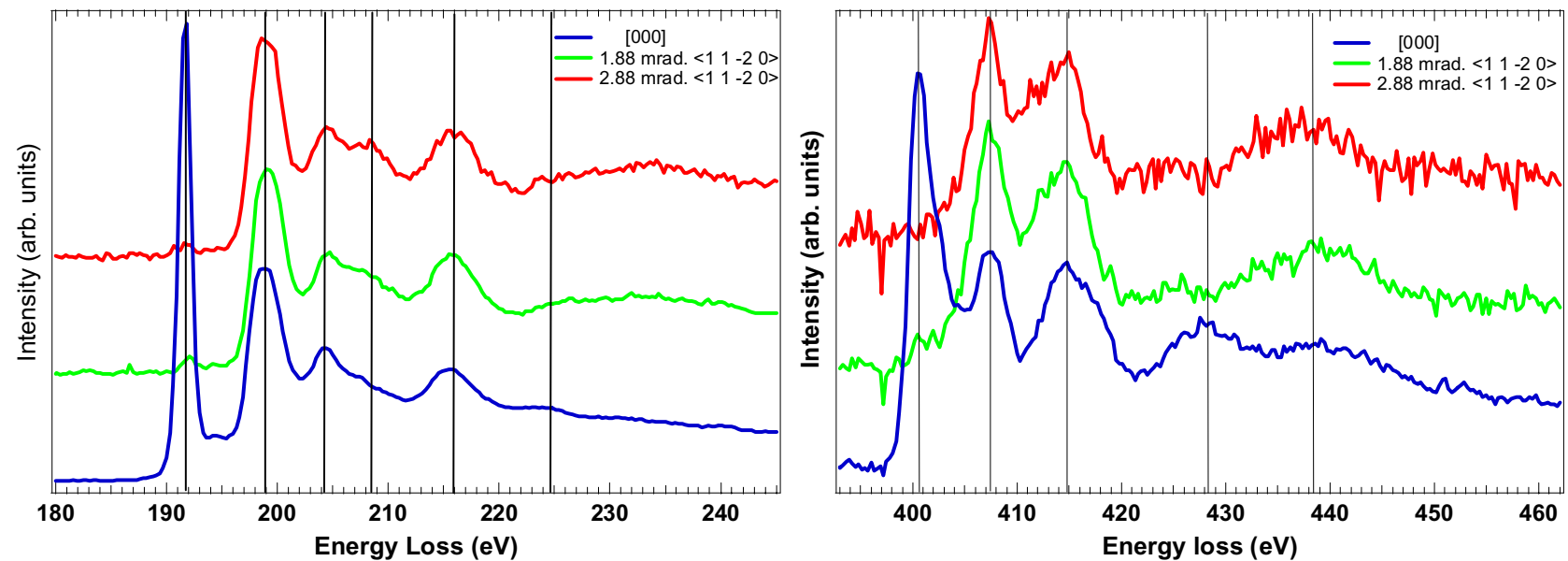

Figure 2. (On the left) B-K edge and (on the right) N-K edge for three scattering vectors marked in Figure 1 (c). 